\title{
Fulminant Progressive Brainstem Encephalitis as Initial Manifes- tation of NeuroBechet's Disease
}

\author{
${ }^{1}$ Internal Medicine Service, Hospital el Bierzo, Spain \\ ${ }^{2}$ Neurology Service, Hospital Universitario Central de Asturias (HUCA), Spain \\ ${ }^{3}$ Neurology Department, Hospital el Bierzo, Spain \\ ${ }^{4}$ Emergency Room Service, Hospital el Bierzo, Spain
}

Tierra Rodríguez $A M, M D^{1 *}$, Fernández Díaz $A, M D^{2}$, Pérez Ruíz $D, M D^{3}$ and López Prada $B, M D^{4}$

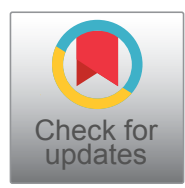

*Corresponding author: Ana María Tierra Rodriguez, MD, Internal Medicine Service, Hospital el Bierzo, 5, Médicos sin Fronteras Av. Ponferrada (León), 24411, Spain, Tel: 0034-9874-55200, Fax: 0098-7455-300

\begin{abstract}
Bechet's disease is a multisystemic process that can mimic many other diseases. Neurological features can be its first-onset symptoms. Brainstem is usually affected, so that a wide differential diagnosis is mandatory. We report the case of a patient who developed a fulminant and rapidly progressive brainstem encephalitis owing to NeuroBechet's disease.
\end{abstract}

\section{Keywords}

Bechet, Encephalitis, Facial Palsy, Pons, Magnetic Resonance Image, Uveitis

\section{Introduction}

Bechet's disease (BD) is a hard-to-diagnose entity. Neurological features can sometimes be the first manifestation of the disease. Its symptoms can be so different, so that a wide differential diagnosis with many neurologic or systemic processes is mandatory. Systemic features can be quite helpful. We report the case of a patient who developed fulminant progressive brainstem encephalitis as onset of Neuro-Bechet's disease. A review of the literature is made also.

\section{Case Description}

A sixty-nine year old male was referred to Emergency Room (ER) in our Hospital. He had antecedents of mild blood hypertension and left facial palsy four years ago. He complained of acute right facial weakness for the last two days. There was no evidence of infectious disease or recent vaccinations. Subsequently, he developed dizziness, nausea and vomiting. At physical examination, right facial palsy was detected. In addition, we found right abducens paresis, right hypoacusis and horizontal left nystagmus.

Blood sample tests requested (ANAS, ANCAS, ACE, Antiphospholipid antibodies, onconeural antibodies, AntiNMO and AntiGQ1b antibodies, RPR, TPHA, HIV and viruses tests) were normal or negative. X-ray chest and Body-CT showed no abnormalities. Brain MRI (Figure 1) showed a high-T2 signal lesion at pons and pontomedullary junction, affecting middle cerebellar peduncles also. MR-spectroscopy showed no evidence of tumoral processes.

An empiric treatment with high-dose steroids was started. The patient improved clinically. So did a new MRI. Nevertheless, his condition worsened after a few weeks, developing painful ulcers and papular lesions on the right forearm.

A new MRI was requested. Worsening of the previous lesions with increased enhancement, mesencephalic and cerebellar edema (crushing the fourth ventricle) were also present (Figure 2). A PET exam showed no evidence of malignancy. A brainstem biopsy was rejected by the reference center of neurosurgery. Skin biopsy showed a palisading neutrophilic granulomatous dermatitis. 

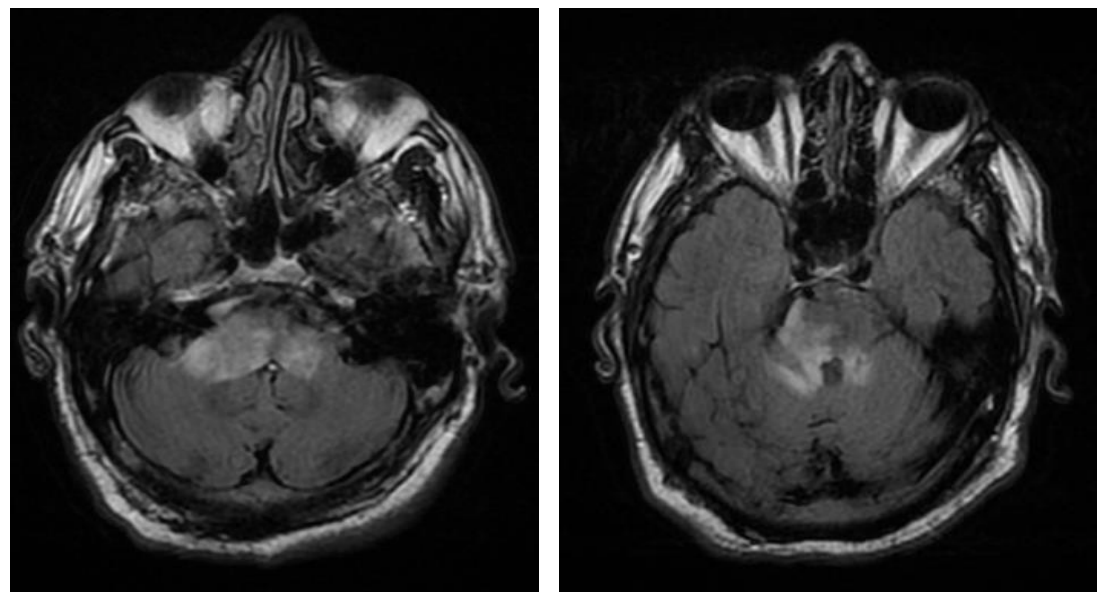

Figures 1: High-intensity signal at pons and both middle cerebellar peduncles.
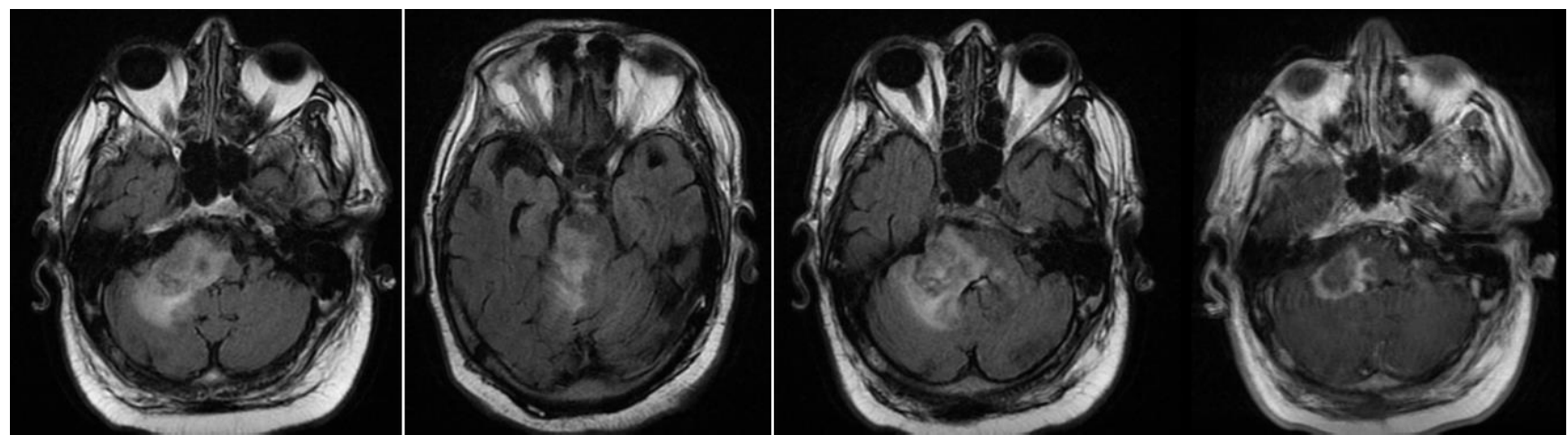

Figures 2: Brain MRI. Axial slices. Hyperintense T2, enhancing lesion and mass effect.

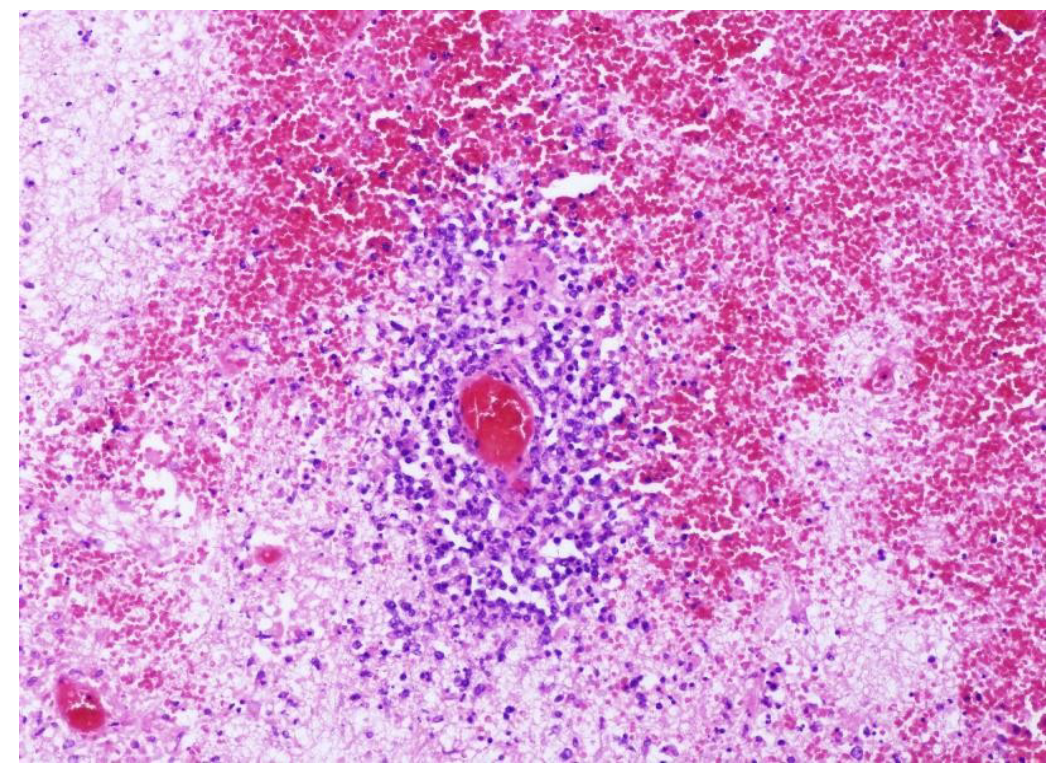

Figure 3: Histopathological features of one of the areas of vasculitis evidenced in the central nervous system (hematoxylin-eosin). There is a small vessel vasculitis with an inflammatory infiltrate invading and destroying the wall. The infiltrate is mainly made of lymphocytes. Prominent accompanying phenomena of hemorrhage and necrosis are also found.

With the diagnosis of possible Bechet's disease (BD) a high dosage treatment with cyclophosphamide was started. Unfortunately, the patient died five days later. Necropsy showed a non-necrotizing, small vessel brainstem vasculitis and an hemorrhagic pontine stroke, re- lated to NeuroBechet's disease (NB), (Figure 3 and Figure 4).

\section{Conclusions}

$\mathrm{BD}$ is a systemic inflammatory disorder. Painful 

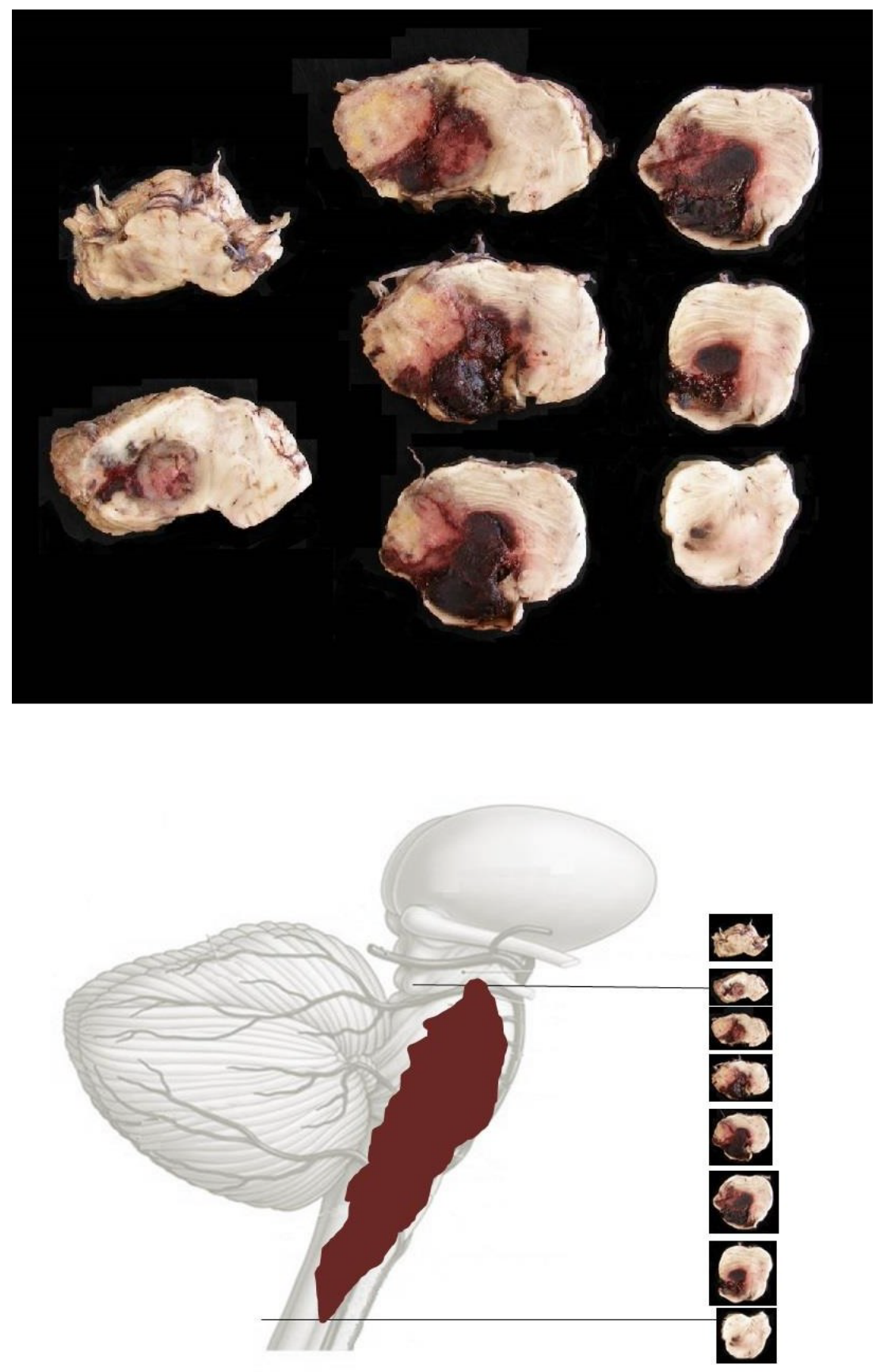

Figure 4: Macroscopic brainstem samples showing hemorrhage and their anatomic location.

and recurrent buco-genital ulcers are the hallmark, although skin lesions, uveitis, vascular events or gastrointestinal disorders can appear also. In rare occasions it can spread to heart, lungs, kidney or peripheral nervous system even.

Young male use to be affected, especially in countries related to the Old Ancient Silk Route. Frequency rates in NB can be quite variable (3-44\% of the patients).

Neurologic disturbances in BD can be classified in two main groups [1]. Parenchymal (meaning $20-60 \%$ of the cases) and non-parenchymal processes (see Table 1). According to literature, brain white matter, brainstem, thalamus and basal ganglia are often impaired.

Clinical disturbances are related to the localization of the lesions [3]. So that, differential diagnosis can sometimes be difficult, especially with Multiple Sclerosis (MS). Sensitivity impairment, optic neuritis, internuclear ophthalmoplegia, limb ataxia or cerebellar dysarthria are more frequent in patient with MS. On the other hand, headache, pseudobulbar speech and cognitive disturbances are more frequent in patients with NB. Other processes like Primary Nervous Central System Lymphoma (PNCSL) need to be excluded [4].

MRI is a fundamental key for NB diagnosis [5]. High-T2 brain signals are found. They usually appear at brain peduncle and pons junction a sugerent feature, pontomedullary junction, thalamic/hypothalamic areas, basal ganglia and cerebellum. On the other hand, subcortical and periventricular areas are not often impaired [6]. In NB, confluent lesions spreading 
Table 1: Clinical features of NB disease.

\begin{tabular}{|l|l|}
\hline Parenchymal forms & Non-parenchymal forms \\
\hline $\begin{array}{l}\text { Brain: Aphasia, cognitive disorders, hemiparesis, sensitivity } \\
\text { disturbances, seizures, psychosis }\end{array}$ & Brain sinus venous thrombosis \\
\hline Optic Neuropathy & Acute Meningeal Syndrome \\
\hline $\begin{array}{l}\text { Brainstem [2]: } \\
\text { Ophthalmoparesis, cranial neuropathy, cerebellar impairment }\end{array}$ & Intracranial Hypertension \\
\hline Spinal Cord & \\
\hline Diffuse or multifocal: Combination of different forms & \\
\hline
\end{tabular}

Table 2: Diagnostic Criteria for NB disease. *ISG: International Study Group Criteria (see Table 3).

\section{Definite disease:}

All the three are required:

1. Positive ISG* for Bechet's Disease.

2. Neurologic syndrome (with objective signs present) known as produced by Bechet disease with typical MRI or CSF findings.

3. No alternative explanation for the neurological findings.

\section{Probable disease:}

1. At least one of these in absence of other better explanation for neurological findings.

2. Neurologic syndrome characteristic (like in the paragraph "definite") and systemic features of BD, but not satisfying ISG criteria.

3. Neurologic syndrome non-characteristic in the context of BD supported by ISG criteria.

Table 3: BD ISG criteria [8]. Main criteria and at least two secondary ones are required. Other diseases need to be excluded.

\begin{tabular}{|l|l|l|}
\hline Mandatory Criteria & Recurrent oral ulcerations & $\begin{array}{l}\text { Minor aphthous, major aphthous or herpetiform ulceration observed } \\
\text { by physician or patient, which recurred at least } 3 \text { times in one } 12 \\
\text { month period }\end{array}$ \\
\hline & Recurrent genital ulceration & Aphthous ulceration or scarring observed by physician or patient. \\
\hline Eye lesion & $\begin{array}{l}\text { Anterior uveitis, posterior uveitis, or cells in vitreous on } \\
\text { slit lamp examination or retinal vasculitis observed by ophthalmologist }\end{array}$ \\
\cline { 2 - 3 } & Skin lesion & $\begin{array}{l}\text { Erythema nodosum observed by physician or patient, pseudo } \\
\text { folliculitis or papulopustular lesions, or acneiform } \\
\text { nodules observed by physician in post-adolescent patients not on } \\
\text { corticosteroid treatment }\end{array}$ \\
\hline & Positive Pathergy test & Behcetine test read by physician 24-48 hours. \\
\hline
\end{tabular}

to thalamus and basal ganglia (the so-called "brainstem plus sign") is [7]. Other diseases like SLE rarely affect the brainstem.

CSF analysis can show abnormalities in $60-80 \%$ of the patients. Mild pleocytosis and raised proteins levels are the most frequent findings, although not specific.

NB Diagnostic criteria [3] were reported in 2013 after an expert meeting (see Tables 2 and Table 3).

Neural biopsy or other histopathologic evidences are not regularly needed, but in atypical cases or exceptional ones like this.

Disease's course is unpredictable, related to the onset clinical features. Worse prognosis is frequently seen in subacute, rapidly progressive cases. Ataxia, bladder incontinence, confusion and less survival are frequently seen in these patients.
Vasculitis is the manifest cause of death. It can affect any caliper and location vessels. Venous impairment happens more often. Brain or kidney arteries are rarely impaired. BD debuts with vascular events in less than $25 \%$ of the cases, mimicking acute strokes.

There are a few cases of patients with NB developing brain hemorrhages without aneurisms or previous vascular abnormalities. Blood Hypertension or hemorrhagic diathesis are the manifest risk factors. Intracranial vasculitis $[9,10]$ can also be another risk factor, although quite unusual (our patient had it).

Concerning therapy, high steroid doses are advocated. Immunosuppressive treatment is considered in severe parenchymal forms. First-line agents include Azathioprine, Methotrexate or Cyclophosphamide. AlphaInterferon, Infliximab or etanercept are required if first line treatment fails or is not well tolerated. 
We report the case of a patient with an atypical, refractory and rapidly progressive form of NB, who developed an exceptional complication (pontine hemorrhagic stroke) due to brain angeitis. Necropsy findings supported our clinical diagnosis.

\section{Acknowledgments}

We would really like to thank Dr. Angel Fernández Flórez for his contribution to the diagnosis of the patient and for providing the pathological and necropsy images.

\section{Conflict of Interests}

No funds have been received for this work. All the authors deny conflict of interests.

\section{References}

1. Mahr A, Belarbi L, Wechsler B, Jeanneret D, Dhote R, et al. (2008) Population-based prevalence study of Behcet's disease: Differences by ethnic originand low variation by age at immigration. Arthritis Rheum 58: 3951-3959.

2. López Bravo A, Parra Soto C, Bellosta Diago E, Cecilio Irazola Á, Santos-Lasaosa S (2017) Neurological manifestations of Behçet's disease: A case description and review of the literature. Reumatol Clin.
3. Chraa Mohamed, Kissani Najib, Lamia Essaadouni (2015) Radiological findings in Behçet disease. Pan Afri Med J 20: 51.

4. Kalra S, Silman A, Akman-Demir G, Bohlega S, Borhani-Haghighi A, et al. (2014) Diagnosis and management of Neuro-Behc, et's disease: International consensus recommendations. J Neurol 261: 1662-1676.

5. Koushun Matsuo, Kei Yamada, Kenji Nakajima, Masanori Nakagawa (2005) Neuro-Behchet disease mimicking brain tumor. Am J Neuroradiol 26: 650-653.

6. Campi M, Tempra A (2003) Casuistry in magnetic resonance. Neuro-Behçet magazine of the private community hospital 6.

7. Liewluck T (2009) A brainstem plus sign in neuro-behçet disease. Inter Med 48: 1483-1484.

8. Toro Giraldo AM, Pinto Peñaranda LF, Velásquez Franco CJ, Márquez Hernández JD (2009) Behçet disease. Rev Colomb Reumatol 16: 97-111.

9. Minju Yeo, Hye-Lim Lee, Minju Cha, Ji Seon Kim, HoSeong Han, et al. (2016) Neuro-Behcet disease presenting as a solitary cerebellar hemorrhagic lesion: A case report and review of the literature. J Med Case Rep 10: 360.

10. Lee WJ, Choi C, Kim JM, Jung KH, Roh JK (2017) Delayed symptomatic intracerebral hemorrhages in neuro-Behcet's disease. J Neurol 264: 394-396. 